\title{
Macromodel-based simulation and measurement of the dynamic pull-in of viscously damped RF-MEMS switches
}

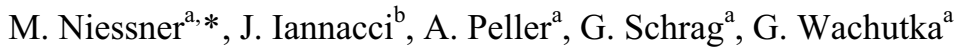 \\ ${ }^{a}$ Institute for Physics of Electrotechnology, Munich University of Technology, Munich, Germany \\ ${ }^{b}$ Fondazione Bruno Kessler (FBK), MEMS Research Unit, Povo di Trento, Italy
}

\begin{abstract}
Three different state-of-the-art approaches are used to generate macromodels of an electrostatically actuated and viscously damped ohmic contact RF-MEMS switch. The capability of the three multi-energy domain coupled models to predict the behavior of the RF-MEMS switch is evaluated w.r.t. white light interferometer and laser vibrometer measurements. The different macromodels show very good agreement concerning the quasi-static measured pull-in/pull-out characteristics. The evaluation of the modeled viscous damping forces demonstrates that tailored physics-based models give significantly better results than simple generic models. However, all approaches fail in capturing the landing phase of the membrane during the dynamic pull-in correctly. Measurements revealed that this is due to the presence of a higher eigenmode that is activated during impact.
\end{abstract}

(C) 2010 Published by Elsevier Ltd. Open access under CC BY-NC-ND license.

Keywords: RF-MEMS switch, macromodel, dynamic pull-in

\section{Motivation}

The simulation of pull-in/pull-out transients is essential for predicting the switching time of a new RF-MEMS switch design, but makes high demands on the utilized model, since in addition to the electrostatic, mechanical and fluidic domains, as well as their nonlinear interactions, also the mechanical contact has to be considered. Furthermore, when multiple coupled energy domains with large mesh deformations have to be analyzed, finite element (FE) models become computationally expensive and difficult to handle, and macromodels with a reduced number of degrees of freedom and, thus, reduced computational expense are necessary to enable a fast simulation of pull-in/-out transients. Naturally, the resulting short computing times in the magnitude of only a few minutes do not come for free: a macromodel is a reduced description of the original problem and the validity of the simulated results strongly depends on the respective approach chosen for the derivation of the coupled macromodel. This paper explores three different approaches [1-3] to generate macromodels of an electrostatically actuated and viscously damped ohmic contact RF-MEMS switch by evaluating the results from macromodel-based simulations w.r.t. measurements preformed with a white light interferometer (WLI) and a laser vibrometer (LV).

\footnotetext{
* Corresponding author. Tel.: +49-89-28923108; fax: +49-89-28923134

E-mail address: niessner@tep.ei.tum.de.
} 
The considered RF-MEMS device is fabricated at Fondazione Bruno Kessler (FBK) [2] and consists of a perforated movable gold membrane suspended by four beams above a fixed ground electrode (cp. fig 1). By applying a voltage beyond the so called pull-in voltage, the membrane collapses onto elevated contact pads (cp. fig. 2) and closes an ohmic contact. The two investigated switch designs have a gap height $g_{0}$ between the membrane and the contact pads of 1.4 and $1.7 \mu \mathrm{m}$, respectively.

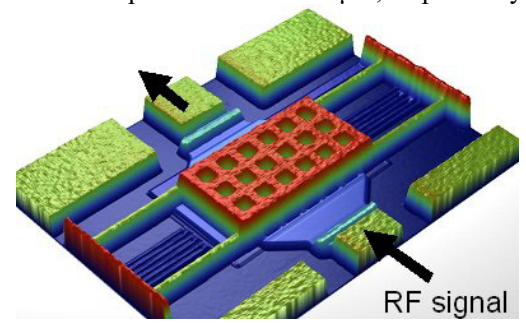

Fig. 1. Measured (WLI) 3D profile of the switch without bias.

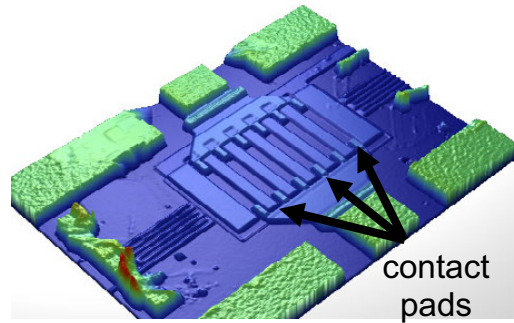

Fig. 2. Measured (WLI) profile of the electrodes and the 12 elevated contact pads. The membrane was removed for this measurement.

\section{Modeling}

In this paper, we focus exclusively on approaches that apply flux-conserving reduced-order and/or compact modeling techniques. The models are formulated in terms of conjugated variables ("across"- and "through"variables) and the generalized Kirchhoffian network theory is used as a theoretical framework for the coupling between multiple energy domains. The simulation of the dynamic pull-in of the switch requires the derivation of four submodels: a mechanical submodel comprising the suspended membrane, an electrostatic one describing the electrostatic field and the attracting forces between membrane and electrode, a model of the viscous damping forces acting on the membrane and a submodel of the mechanical contact between the membrane and the contact pads.

In the approach presented by Schrag and Niessner [1] (abbr. "MLM") various techniques are applied to generate macromodels of the different domains. A superposition of mechanical eigenmodes is used to model the dynamics of the suspended membrane. The electrostatic submodel is derived from a Lagrangian energy functional formulated in terms of the modal amplitudes. The viscous damping forces are modeled by the mixed-level approach presented in [4], i.e. the Reynolds equation is evaluated in a distributed finite network model and combined with compact models, which account for perforations and the outer boundary. The contact force at a specific node $n$ is modeled by a dissipative term that accounts for the losses during contact and a contact stiffness that accounts for the deformation stiffness of the pads:

$$
F_{\text {contact }, n, i}(t)=d_{\text {contact }, i} \dot{q}_{i}+k_{\text {contact }}\left(h_{n}(\bar{q}, t)-g_{0}\right)
$$

Here, $\bar{q}$ denotes the vector of modal amplitudes, $\dot{q}_{i}$ the velocity of the $i$-th mode, $h_{n}(\bar{q}, t)$ the local displacement at the $n$-th node and the parameters $\boldsymbol{d}_{\text {contact }, i}$ and $\boldsymbol{k}_{\text {contact }}$ the lumped contact loss and stiffness. By means of this formulation, the approach is able to simulate bouncing during the landing of the membrane.

The approach presented by Iannacci [2] (abbr. "Iannacci") uses solely lumped models: four lumped linear beam models for the suspensions, a rigid plate model with both translational and rotational degrees of freedom for the membrane, and a lumped capacitor for the electrostatic domain. The viscous damping is calculated through a modified Reynolds equation that accounts also for perforations. The contact model is included by introducing a linear force with a contact stiffness similar to $\boldsymbol{k}_{\text {contact }}$ in equation 1.

A lumped element approach is also implemented in the commercial software COVENTORWARE Architect3D ${ }^{\mathrm{TM}}$ (abbr. "Architect3D"). Here, a high-order non-linear beam model is used for the suspensions. The membrane is modeled by the mixed interpolation of the tensorial components approach (MITC) [3], the electrostatic domain by a lumped capacitor, and the viscous damping by a generic lumped submodel that is based on the Reynolds equation without any modifications for geometrical specifics. For complex geometries, the manual recommends to perform a $\mathrm{FE}$ analysis of the fluidic problem and to extract a mathematically reduced model for the simulation in Architect3D. In this work, the lumped Reynolds-based model is used. For the mechanical contact, an available standard model is included in the simulation which is, however, not described in detail within the manual. 


\section{Results}

The evaluation of the three fully coupled macromodels was performed in four steps. First, the fabrication-induced residual stress in each mechanical submodel was calibrated, so that the simulated resonance frequencies corresponded to the measured one of $14.7 \mathrm{kHz}$. Second, the quality of the modeled coupling between the electric and the mechanical submodels was validated by comparing the quasi-static pull-in/-out characteristics of the switch to measured data. Third, the performance of the fully coupled macromodels was evaluated by measured pull-in/-out transients, and, fourth, a detailed analysis of the landing phase of the membrane during the pull-in was performed.

For the quasi-static pull-in/-out characteristics, the switch was actuated by a $70 \mathrm{~V}$ pp (peak-to-peak) zero mean value triangular waveform at $20 \mathrm{~Hz}$. Figure 3 shows that the pull-in of the membrane occurred at $30.5 \mathrm{~V}$ and the release at $24 \mathrm{~V}$. All three models were able to reproduce the pull-in characteristic correctly, but calculated a too early release (cp. fig. 3; the model of Iannacci was omitted for the sake of clarity). The incorrect calculation of the release is due to the fact that no dielectric charging, no adhesion forces or other contact-related phenomena were yet considered in any of the contact submodels.

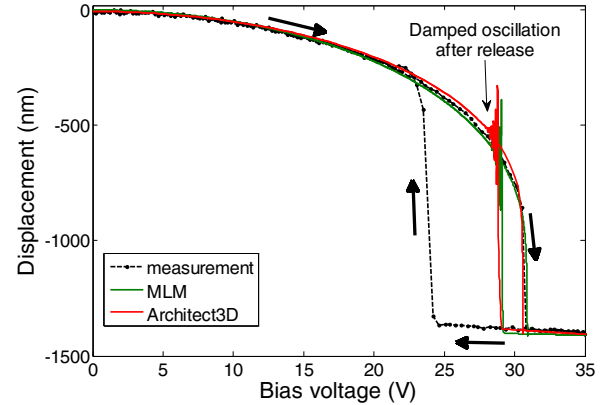

Fig. 3: Quasi-static measured (WLI) and simulated pull-in/-out of the membrane $\left(g_{0}=1.4 \mu \mathrm{m}\right)$. The damped oscillation after release was not recorded because of the low sampling rate used in this measurement.

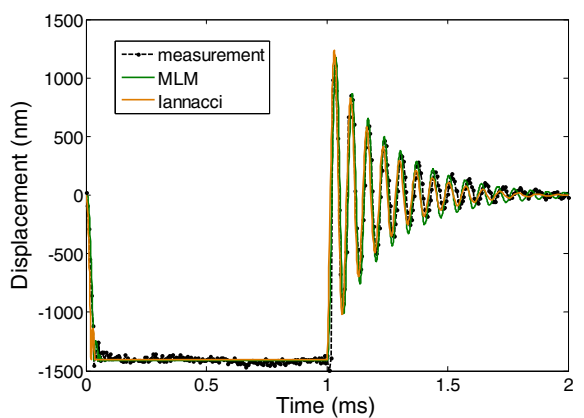

Fig. 4: Measured (WLI) and simulated pull-in/-out of the membrane $\left(g_{0}=1.4 \mu \mathrm{m}\right)$ for a rectangular waveform at $500 \mathrm{~Hz}$ : amplitudes $36 \mathrm{~V}$ (on) and $0 \mathrm{~V}$ (off). $50 \%$ duty-cycle.

The pull-in/-out transients were measured by actuating the switch with a rectangular voltage waveform with an amplitude of $36 \mathrm{~V} \mathrm{pp}$. Due to their tailored descriptions of the fluidic damping taking also perforations adequately into account, the MLM and Iannacci models reproduced the damped oscillation after the release of the membrane accurately and showed a very good agreement with the measurements (cp. fig. 4). In contrast, Architect3D overestimates the damping forces although we used a so-called "best-fitted" damping model (cp. fig. 5). This is certainly due to the missing physics-based consideration of the perforation holes.

To get a highly time-resolved measurement of the landing phase of the membrane during dynamic pull-in, we used a LV with a sampling rate of $2.56 \mathrm{MHz}$ and measured the response of the switch to an actuation with a rectangular voltage waveform of $35 \mathrm{~V}$ pp. Figure 6 shows the displacement calculated from measured velocity data vs. simulated results. All simulations differ from the measured data. A detailed view on the measured displacement, however, gives evidence of several different superimposed vibrations involved in the contact process, which is proven by a FFT analysis of the landing phase (cp. fig. 7) that confirms the presence of two modes at 87 and $218 \mathrm{kHz}$. The highly increased frequencies, compared to the eigenfrequency of $14.7 \mathrm{kHz}$, are reasonable because the high contact stiffness now couples with the stiffness of the suspended membrane. A FFT analysis of the release phase (cp. fig. 8) shows on the other hand the presence of two modes at 14.7 and $136 \mathrm{kHz}$. A FE modal analysis shows that the structure has indeed a symmetric eigenmode at $130 \mathrm{kHz}$. Furthermore, when the switch is actuated with a $25 \mathrm{~V}$ pp rectangular waveform, i.e. the pull-in does not occur, only the mode at $14.7 \mathrm{kHz}$ is present (cp. fig. 8).

These experimental findings lead to the assumption that, during the first impact of the membrane, most of the kinetic energy of the fundamental mode at $14.7 \mathrm{kHz}$ is transferred to the next higher symmetric mode at $136 \mathrm{kHz}$. This would imply that the landing phase can only be captured correctly when multiple and also higher modes are considered in the macromodel and when the coupling of these modes during impact is implemented. 


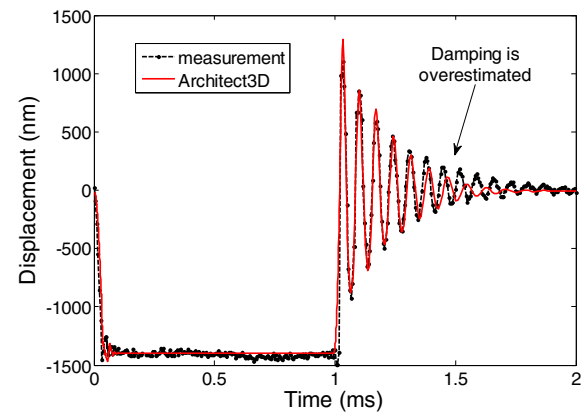

Fig. 5: Measured (WLI) and simulated pull-in/-out of the membrane $\left(g_{0}=1.4 \mu \mathrm{m}\right)$ for a rectangular waveform at $500 \mathrm{~Hz}$ : amplitudes $36 \mathrm{~V}$ (on) and $0 \mathrm{~V}$ (off). $50 \%$ duty-cycle.

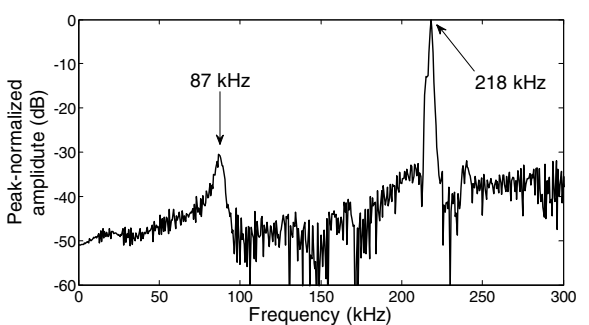

Fig. 7: Frequency spectrum of the measured (LV) landing phase $(0 . .2 \mathrm{~ms})$ of the membrane $\left(g_{0}=1.7 \mu \mathrm{m}\right)$. Voltage $35 \mathrm{~V}$, frequency $250 \mathrm{~Hz}$. A Hanning window was used for the FFT in MATLAB ${ }^{\text {TM }}$.

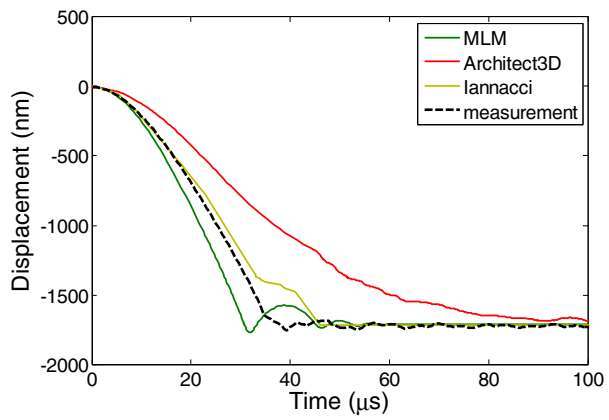

Fig. 6: Measured (LV) and simulated landing of the membrane $\left(g_{0}=1.7 \mu \mathrm{m}\right)$ for a rectangular waveform at $250 \mathrm{~Hz}$ : amplitudes $35 \mathrm{~V}$ (on) and $0 \mathrm{~V}$ (off). $50 \%$ duty-cycle. Only the first $100 \mu$ s are shown.

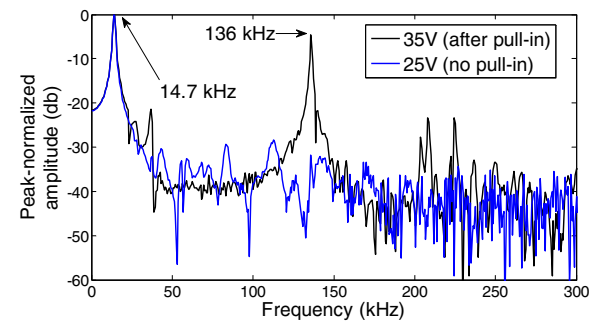

Fig. 8: Frequency spectrum of the measured (LV) release phase $(2 . .4 \mathrm{~ms})$ of the membrane $\left(g_{0}=1.7 \mu \mathrm{m}\right)$. Voltages $35 \mathrm{~V}$ and $25 \mathrm{~V}$, frequency $250 \mathrm{~Hz}$. A rectangular window was used for the FFT in $\mathrm{MATLAB}^{\mathrm{TM}}$.

\section{Conclusion}

Three different approaches [1-3] were used to generate macromodels of an RF-MEMS switch and to simulate the dynamic pull-in/-out of the membrane. The electric and mechanical coupled submodels derived by the three approaches showed very good agreement w.r.t. the quasi-static pull-in/out characteristics. Although a lot of work was done in this field [4], the predictive simulation of fluidic damping remains a challenging task. The strong influence of the topography of the moving structure on the fluidic damping prohibits the use of generic models and requires a tailored modeling strategy. The modeling of the landing of the membrane during dynamic pull-in is identified as an issue for further research as no macromodel was able to capture the physics in this phase correctly. The FFT of the measured landing phase showed that a higher eigenmode is involved and needs to be considered in a macromodel as well as the coupling between the modes occurring during impact. Without the inclusion of higher modes, the only option for getting results with a minimum of reasonability is to emulate the impact-induced transfer of kinetic energy by introducing a loss mechanism by means of a lumped dissipative description like in eq. 1 or by using strong numerical damping whilst pull-in simulation.

\section{References}

[1] Niessner M, et al. Non-linear model for the simulation of viscously damped RF-MEMS switches at varying ambient pressure conditions, Proc. Eurosensors XXIII; 2009, p. 618-621.

[2] J. Iannacci, at al. Experimental Validation of Mixed Electromechanical and Electromagnetic Modeling of RF-MEMS Devices Within a Standard IC Simulation Environment, Journal of Microelectromechanical Systems, vol.19, no.3; 2010, p. 526-537.

[3] Coventor, Inc. Coventorware Architect Version 2008.10 Reference; 2008.

[4] Schrag G, Wachutka G. Physically based modeling of squeeze film damping by mixed-level system simulation. Sensors and Actuators A, vol. 97-98; 2002, p. 193-200. 